\title{
VARIABLES QUE INCIDEN EN BAJA PRODUCTIVIDAD EN MANO DE OBRA
}

\section{VARIABLES QUE INCIDEN EN BAJA PRODUCTIVIDAD EN MANO DE OBRA}

\author{
${ }^{a}$ Esp.Pedro Nel angarita Uscategui, Esp. ${ }^{b}$ Leandro Ovallos Manosalva \\ ${ }^{a}$ Docente Ocasional, Grupo de Investigación GIGMA, Ocaña, Colombia, \\ pnangaritau@ufpso.edu.co \\ ${ }^{\mathrm{b}}$ Docente Ocasional, Grupo de Investigación GIGMA, Ocaña, Colombia, \\ lovallosm@ufpso.edu.co
}

\begin{abstract}
Resumen: La baja productividad ha sido objeto de estudio por parte de todo tipo de industrias y empresas, especialmente en esta época donde la competencia obliga a que los niveles de productividad sean cada vez más altos, sin embrago en la industria de la construcción son pocos los estudios que en materia de este tema se hallan realizado. Referentes internacionales y nacionales concluyen que los índices de productividad en las actividades de la obra es eminente solo el 50\% de la jornada es laboral. El municipio de Ocaña, Norte de Santander no es ajeno a estos estándares, sin embargo no se tienen en cuenta estos índices en la planeación de los diferentes proyectos de construcción y no se cuenta con información para conocer las variables que influyen en la baja productividad; de allí la importancia de desarrollar estudios que permitan la identificación de variables que inciden la baja productividad de las diferentes actividades de construcción en la ciudad de Ocaña y el análisis necesario para calcular los índices de productividad en nuestra región. Estas variables de estudio serán un factor fundamental para alcanzar el éxito de los proyectos.
\end{abstract}

Palabras clave: Productividad, recursos, trabajo, tiempo, variables de incidencia

Abstract: The study allowed us to determine which teaching practices are not aligned to the pedagogical model of the University, which is the Social Constructivism, so it is necessary to establish an improvement plan aimed at strengthening teaching practices and that these in turn were relevant with the pedagogical model Low productivity has been studied by all types of industries and companies, especially in this era where competition requires that productivity levels are higher and higher, without clutch in the construction industry are few studies that regarding this issue are made. international and national benchmarks conclude that productivity rates in the activities of the work is eminent only $50 \%$ of the day's work. The municipality of Ocaña, Norte de Santander is no stranger to these standards, however not taken into account 
these indices in planning various construction projects and no information is available to identify variables that influence low productivity; hence the importance of developing studies to identify variables that affect the low productivity of the different construction activities in the city of Ocaña and the need to calculate productivity indices in our region analysis. These variables study will be a key success factor of the project.

Keywords: Productivity, resources, work, time, incidence variables.

\section{INTRODUCCIÓN}

La educación como proceso de socialización, es tan antigua como el mismo ser humano; como toda creación natural ha sido un tema de gran debate. De allí que históricamente muchos estudiosos se han ocupado de reflexionar sobre la misma, pero quizás tal reflexión se intensifica y sistematiza al ritmo del desarrollo científicotécnico.

Al transcurrir los años, se han generado diversas teorías, enfoques, modelos pedagógicos que dan razón a un momento histórico, cultural y social; estos constituyen parámetros específicos relacionados con los procesos de enseñanza- aprendizaje ajustados a las características de las instituciones y del medio social en la cual se desarrollan dichas instituciones.

Es de importancia, en este momento, entender que el que el saber pedagógico no se refiere solo a lo que se ha pensado en torno a la educación, sino que está caracterizado sobre todo por la reflexión que el docente está haciendo en forma constante a partir o con respecto a lo que realiza en el procesos de enseñanza

Desde la perspectiva anterior y teniendo en cuenta que las reflexiones en torno a los procesos pedagógicos, son constantes y diversos, podría decirse que en la actualidad las crisis educativa, no se resuelve eligiendo entre la variada oferta de estrategias y metodologías las más adecuadas, dejando de lado métodos que en este momento son llamados arcaicos, tradicionales, en el sentido que muchos manifiestan que no sirven y que hay que aplicar nuevas formas de enseñanza, afirmación arriesgada, pues quizás no existe procedimiento que sea más adecuado que el otro, mejor que el otro; de tal manera que para estar en lo correcto se tuviera que desechar todo lo que individualmente se considere inadecuado; lo que sí se puede hacer es generar discusión alrededor de este tema trascendental y por supuesto preguntarse: ¿por qué unos métodos suelen ser más adecuados que otros? ¿Por qué se manifiesta abiertamente que la cátedra magistral ya no sirve y que hay que reemplazarla por otras formas de impartir el saber? ¿En qué consiste la transformación curricular?

Como lo establece Murcia, citado por Prieto (2002).

El análisis de la realidad concreta y cotidiana de las salas de clases, permite visualizar la existencia de una combinación de elementos que interactúan entre sí. Los efectos de esta interacción, no sólo a veces apuntan hacia propósitos divergentes, sino que, en muchos casos, aparecen contradictorios respecto de lo que supuestamente deberían ser los objetivos del proceso. La interacción implica, necesariamente la comprensión 
de significados y la interpretación recíproca de los actos propios y en la que la cotidianidad es permanentemente definida y enmarcada en un contexto físico, institucional, histórico y cultural. Estos contextos afectan de manera indiscutible los logros del aprendizaje, por lo que resulta casi ingenuo atribuir relaciones de causalidad entre medios o métodos del profesor y rendimiento, pues son muchos otros los elementos y aspectos que están interactuando e incidiendo en los efectos o resultados del proceso (p.73)

En consecuencia de los aspectos anteriormente citados, surgen los modelos pedagógicos, los cuales según Bernal (2010).

Son representaciones ideales del mundo de lo educativo para explicar teóricamente su hacer. Dichos modelos son dinámicos, se transforman y pueden, en determinado momento, ser aplicados en la práctica pedagógica. Por lo tanto el modelo pedagógico implican los contenidos, los modos de la enseñanza de acuerdo al desarrollo de los niños y niñas y las características de la práctica docente que pretende lograr aprendizajes y se concreta en el aula. Se considera también el modelo pedagógico como un instrumento de la investigación de carácter teórico creado para reproducir idealmente el proceso enseñanza aprendizaje, paradigma que sirve para entender, orientar y dirigir la educación. Se hace evidente la diversidad de conceptos determinantes asociados a la definición de modelo pedagógico (p.4).

Por otro lado de acuerdo a Gago (2002) "un modelo pedagógico, es una representación arquetípica o ejemplar del proceso de enseñanza-aprendizaje, en la que se exhibe la distribución de funciones y la secuencia de operaciones en la forma ideal, que resulta de las experiencias recogidas al ejecutar una teoría del aprendizaje" (p.2)

El estudio de los modelos pedagógicos permite a los docentes tener un panorama de cómo se elaboran los programas, de cómo operan y cuáles son los elementos que desempeñan un papel determinante en un programa o en una planeación didáctica. En algunos de ellos los profesores pueden ver claramente los elementos más generales que intervienen en una planeación didáctica, así como las relaciones de antecedente y consecuente que guardan entre sí. De allí que Porlan (1993) manifiesta:

Para poder identificar un modelo pedagógico se necesita conocer sus características fundamentales que, según Porlan surgen al responder tres preguntas esenciales: ¿Que enseñar? Es decir, qué contenidos, en qué secuencias y en qué orden, su enseñabilidad y relevancia; ¿Cómo enseñar? Se refiere a los métodos, medios y recursos. Aquí adquieren un Valor relevante los estilos de enseñanza de los maestros y de aprendizaje de los estudiantes: ¿Qué y cómo evaluar? Referido no sólo a los momentos, sino también a los instrumentos de comprobación y a los contenidos previstos desde el inicio del proceso. En este aspecto también adquieren importancia los estilos de enseñanza y aprendizaje (p.15). 
enfoque, una metodología y una evaluación; es indispensable conocer también qué percepción tienen de estos tres elementos los estudiantes, los mismos docentes para con ello facilitar y aclarar qué modelo pedagógico puede ser pertinente de acuerdo a las características curriculares, colectivas y sociales, además permite concebir de manera clara la practica educativa.

Según Astolfi, (1997) hay tres modelos predominantes en la enseñanza: "transmitivo, de condicionamiento y constructivista, que sirven -explícita o implícitamente-, como base para las prácticas y estilos de los maestros. Cada uno dispone de una lógica y de una coherencia que habrá de caracterizarlo, pero sobre todo, cada uno responde a diferentes situaciones de eficiencia" (p.34)

\section{Continua diciendo Astolfi et al (1997)}

El modelo transmitivo o tradicional se refiere principalmente a la elaboración de un programa de estudios. Los elementos que presentan son mínimos, ya que no se hacen explícitas las necesidades sociales, la intervención de especialistas, las características del educando, ni tampoco se observan las instancias de evaluación del programa de estudios. El esquema es muy sencillo. En él destacan los cuatro elementos siguientes:

- El profesor, que es el elemento principal en el modelo tradicional, ya que tiene un papel activo: ejerce su elocuencia durante la exposición de la clase, maneja numerosos datos, fechas y nombres de los distintos temas, y utiliza el pizarrón de manera constante.
- El método: Se utiliza cotidianamente la clase tipo conferencia, abundantes apuntes, la memorización, y la resolución de los cuestionarios que presentan los libros de texto.

- El alumno, que en este modelo no desempeña una función importante, pues su papel es más bien receptivo, es decir, es tratado como objeto del aprendizaje y no se le da la oportunidad de convertirse en sujeto del mismo.

- La información o contenido se presenta como temas, sin acotar la extensión ni la profundidad con la que deben enseñarse. De esta manera, algunos profesores desarrollan más unos temas que otros creando distintos niveles de aprendizaje en grupos de un mismo grado escolar.

Este Modelo concibe la enseñanza como una actividad artesanal y al profesor como un artesano cuya función es explicar claramente y exponer el conocimiento a los estudiantes de manera progresiva. $\mathrm{Si}$ se presentan errores, son atribuibles al alumno por no adoptar la actitud esperada. Dentro de esta concepción educativa se pueden distinguir dos enfoques de la práctica del docente:

Astolfi et al (1997.p 23) el primero es un enfoque enciclopédico, en el que el profesor es un especialista lleno de información; la enseñanza es una pura transmisión que al final se resume en la acumulación de conocimientos y no se hace distinción entre saber y saber enseñar.

El segundo enfoque es el comprensivo, en el que el profesor es un intelectual que comprende lógicamente la estructura de la materia pero sólo la transmite. En ambos 
enfoques se resta importancia al conocimiento pedagógico que no esté relacionado con las disciplinas en su modo de transmisión y presentación, ni al conocimiento que se deriva de la experiencia práctica como docente. Se trata de un aprendizaje basado en la teoría.

En resumen, en esta perspectiva el aprendizaje es la simple comunicación entre emisor (maestro) y receptor (alumno) y se ignora el fenómeno de comprensión y el proceso de la relación con sentido de los contenidos.

Siguiendo a Astolfi, et al (1997 p.13) el modelo de condicionamiento o pedagogía (conductista), está basado en los estudios de Skinner y Pavlov sobre aprendizaje; se enfatiza en los medios necesarios para llegar a un comportamiento esperado y verificar su obtención.

Finalmente, el Modelo Constructivista o de perspectiva radical, concibe la enseñanza como una actividad crítica, y al docente como a un profesional autónomo que investiga reflexionando sobre su práctica. Este modelo difiere de los anteriores en la forma como se maneja el concepto de error: es un indicador que permite hacer análisis de los procesos intelectuales que ocurren al interior de quien aprende. Para el constructivismo, aprender es arriesgarse a errar (ir de un lado a otro), y muchos de los errores cometidos en situaciones didácticas deben considerarse como momentos creativos. Para el constructivismo la enseñanza no es una simple transmisión de conocimientos; es una tarea de organización de métodos de apoyo y situaciones de aprendizaje que permiten a los alumnos construir su propio saber. No se aprende sólo registrando en el cerebro. Se aprende construyendo la propia estructura cognitiva.

Desde las apreciaciones anteriores, la Educación superior debe garantizar una educación de calidad; para ello es fundamental que los programas que se oferten se fundamenten en currículos que ofrezcan a los estudiantes el desarrollo de competencias que se ajusten a las necesidades de la sociedad.

Indudablemente, uno de los componentes básicos en la calidad educativa, son las prácticas docentes, estas, son variables fundamentales en los procesos académicos, ya que de las buenas prácticas docentes depende la formación de los futuros profesionales, formación que debe estar de la mano con lo que la sociedad exige.

Las prácticas docentes no pueden estar desarticuladas con el modelo pedagógico que las universidades adoptan teniendo en cuenta el contexto, dicho modelo pedagógico debe verse reflejado a través de los métodos, técnicas y metodologías aplicadas a través del desarrollo didáctico de la asignatura, por supuesto no se puede dejar de lado la evaluación, la cual también debe estar correlacionada con el modelo pedagógico; de esa manera, el proceso de enseñanza- aprendizaje y la calidad académica sería más pertinente.

Desde lo anterior, se hizo importante realizar un estudio que permitiera determinar y conocer las prácticas docentes y su relación con el modelo pedagógico de la Universidad, en este caso, con el modelo pedagógico de la Universidad Francisco de Paula Santander Ocaña, el cual corresponde al modelo Constructivista Social. 
El Proceso investigativo dejó concluir que hace indispensable iniciar un proceso de formación y autoformación por parte de los docentes que ayude a transformar al docente, ya que se consideró que el modelo pedagógico de la Universidad en primera mediada, no es conocido, solo se identifica el nombre, pero, se desconoce las bases epistémicas del mismo, sus características, su didáctica, en fin no hay claridad al respecto, en consecuencia, las clases son una mezcla de distintos modelos.

La formación no es tarea fácil ya que esto exige involucrar una serie de cambios en los diseños curriculares, en el diseño de la institución como eje fundamental del proceso, modos de evaluar la docencia y al docente, por lo que éste proceso implica que la Institución trace un plan de mejoramiento institucional y docente a largo y corto plazo.

De tal manera, pues, lo que hay que emprender es la reflexión pedagógica a través de una forma holística, resultando conveniente no buscar fórmulas de acuerdo a posibles modelos y / o estrategias metodológicas sino más bien entender lo pedagógico como una toma de decisiones críticas acerca del proceso mismo de la educación convirtiendo al docente, acá, como un eje fundamental; dicho de otra manera, el docente se convierte en un actor principal en el proceso pues es él quien define y articula las distintas metodologías que permiten mejorar los procesos académicos.

Indiscutiblemente, desde la mirada anterior, el saber pedagógico, no puede limitarse a un saber instrumental acerca de cómo manejar y aplicar un modelo curricular adoptado o en vías de ser adoptado e impuesto desde la instancia superior tal como lo exige la actual racionalidad instrumental y funcional, de acuerdo con los lineamientos de la tecno ciencia; estas apertura ha implicado que la modernización exigida se ha entendido como la superación acrítica del pasado, al respecto Bedoya (2011) indica que:

Hay que adoptar necesariamente el nuevo esquema impuesto ya que estas serán las nuevas reglas de juego, por lo tanto deseosos de que todo cambie o de que la corriente de cambio los envuelva se ha cambiado por cambiar, sin entender porque hay que hacerlo, sin asumir el reto de la reflexión (p.45).

Por otro lado y respecto a las anteriores apreciaciones, no se puede dejar de lado y restarle importancia a la llamada "revolución cognitiva", es valioso destacar que en este siglo, la comprensión del aprendizaje se debe ver reflejada en una mayor comprensión de la naturaleza social del aprendizaje, del impacto del contexto en los procesos de pensamientos de los educando La productividad puede definirse como la relación entre la cantidad de bienes y servicios producidos y la cantidad de recursos utilizados y es indispensable para evaluar el rendimiento de las máquinas, los equipos de trabajo y los obreros. En la industria de la construcción la productividad de los diferentes recursos empleados es de gran importancia en el desarrollo exitoso de un proyecto, ya que su dinámica, es un motor que impulsa permanentemente el progreso de la obra, sin embargo, en la construcción el trabajo del recurso humano en general es poco eficiente, convirtiéndose en un factor crítico para el desarrollo del proyecto, teniendo en cuenta que un gran 
porcentaje de la jornada laboral es improductiva por parte de los frentes de trabajo para elaborar las actividades en una obra, esto se ve reflejado en la línea base del proyecto, en cuanto al alcance, tiempo, costo y calidad.

Algunos autores, como (Borcherding, 1976) establecen que "al contrario de lo que ocurre en la industria en general, en la industria de la construcción las actividades bien organizadas y planeadas, que permiten que los trabajadores sean productivos, traen consigo satisfacción en el trabajo; en tanto, la insatisfacción acompaña a proyectos pobremente planeados". Según (Tucker, 1988) en un estudio realizado para el caso del trabajador de la construcción en Estados Unidos concluye que "solo el $40 \%$ de la jornada de trabajo resulta ser productiva" evidenciando los bajos índices de eficiencia que serán determinantes en el cumplimiento de la programación planeada; adicionalmente (Martínez y Alarcón,1988) establecen que "el trabajo del recurso humano en la construcción en Chile en general, es poco eficiente, más del $50 \%$ del tiempo total de trabajo es destinado a trabajo no contributario a la producción", la disminución de la productividad del recurso humano podrían ser explicadas con las opiniones de (Maloney y Federle 1993) quienes perciben que el paradigma Tayloriano de división del trabajo $\mathrm{y}$ especialización no rinde frutos en la organización de las empresas constructoras. De acuerdo a lo anterior la necesidad de desarrollar estudios que clarifiquen resultados óptimos se hace importante para este tema tan trascendental.
En los países del primer mundo se han realizado estudios sobre la productividad, pero aun en ellos, el enfoque hacia el campo de la construcción es escaso, en países como el nuestro estos estudios son casi nulos, de allí la importancia de apoyar la investigación en esta área, aplicando metodologías que afiancen el conocimiento respecto a estas variables en el sector de la construcción. Los procesos de calidad en la productividad del recurso humano juegan un papel importante según (Ishikawa, 1986) "De manera somera calidad significa calidad del producto. Más específico, calidad es calidad del trabajo, calidad del servicio, calidad de la información, calidad de proceso, calidad de la gente, calidad del sistema, calidad de la compañía, calidad de objetivos, etc". De acuerdo a este aparte los procesos de calidad son fundamentales en la productividad, si conocemos las causas que generan la baja producción, podremos proponer alternativas para satisfacer las necesidades de los clientes, esto trae como consecuencia que surjan en las organizaciones como elemento de gran importancia, tener altos niveles de calidad en el recurso humano.

\section{METODOLOGÍA}

La investigación que sirvió de base para la elaboración de éste artículo, se orientó bajo un enfoque cualitativo. Donde los contextos estructurales y situaciones estudiadas identifican la naturaleza profunda de las realidades y su sistema de relaciones. A partir de este acercamiento metodológico en la ejecución del presente estudio, las actividades que lo materializaron, se enuncian a continuación: 
Se realizó una identificación de variables que insidian en la baja productividad en las actividades de construcción, para ello se tomo bibliografía de algunos autores que han tratado este tema se encontró en un manual de Estimator's construction ManHour Manual de John S.Pane donde se plasmaban un numero de variables las cuales se tuvieron como base para el estudio, también se tuvo en cuenta el aporte científico del artículo de Luis Fernando Botero Botero titulado: Análisis de rendimientos y consumos de mano de obra en actividades de construcción de la Universidad EAFIT y entrevistas a profundidad a profesionales con injerencia directa en este campo. Luego se tomaron 10 obras de construcción cuya muestra representativa fue la mitad más uno Las restricciones o limitaciones.

Luego de este paso se realizó encuesta tipo cerrada para la estimación de juicios con respecto al valor de incidencia de las variables de más baja productividad en las actividades en obra en Ocaña a diferente personal en los que se cuenta: Maestros, oficiales, obreros. La finalidad de la encuesta es obtener las variables de mayor incidencia en el estudio. Para tomar un número adecuado de encuestados y de acuerdo a experiencias investigativas se tomara para el desarrollo de la misma una formula estadística para una población finita se muestra a continuación:

$$
n=\frac{\mathrm{N} * \mathrm{Za}^{2} * \mathrm{p} * \mathrm{q}}{\mathrm{d}^{2} *(N-1)+\mathrm{Za}^{2} * \mathrm{p} * \mathrm{q}}
$$

$\mathrm{N}=$ Total de la población.

$\mathrm{Za}=$ Confiabilidad o seguridad de la muestra.

$\mathrm{p}=$ Proporción esperada.

$\mathrm{q}=1$ - Proporción esperada.

$\mathrm{d}=$ Precisión.
Luego de desarrollada la encuesta se validó la confiabilidad de la misma, se utilizó el modelo del coeficiente de alfa Combrash para medir dicha confiabilidad que para proyectos de investigación este coeficiente debe estar entre 0,7 a 0,8 si el resultado de encuestas realizadas no se ajusta a este rango se tendrá que realizar más encuestas para el estudio. La fórmula de alfa Combrash se muestra a continuación:

$\alpha=\left[\frac{k}{k-1}\right] *\left[1-\left(\frac{\sum_{i=1}^{k} S i^{2}}{S t^{2}}\right)\right]$

$S i^{2}=$ varianza del item $i$.

$S t^{2}=$ varianza de los valores totales

$k=$ numero de preguntas o items.

Luego de validar la confiabilidad de la encuesta cerrada se procesan los datos, se obtienen variables que inciden en la baja productividad en diferentes actividades de construcción, para tomar las variables más representativas se toma un modelo estadístico aplicando el principio de Pareto que es también conocido como la regla del 80-20 y recibe este nombre en honor a Vilfredo Pareto, quien lo enunció por primera vez. El principio dice que el $20 \%$ de una acción producirá el $80 \%$ de los efectos, mientras que el $80 \%$ restante sólo origina el $20 \%$ de los efectos. De acuerdo a este principio se evalúan los diferentes ítems causales de la baja productividad en las obras de Ocaña.

De acuerdo al principio de Pareto se identifican las principales variables que causan los problemas de baja productividad en las diferentes obras de construcción en Ocaña para luego determinar las posibles sub causas se emplea el diagrama de 
ISHIKAWA que una representación gráfica que organiza de forma lógica y en orden de mayor importancia las causas potenciales que contribuyen a crear un efecto o problema; creado por Kaoru Ishikawa en la Universidad de Tokio en 1943 para su uso por los círculos de Calidad. En nuestro caso mejorando la calidad de la productividad en obras de Ocaña.

Por ultimo se desarrollan las posibles soluciones, se toman las sub causas identificadas y se toma juicio de los expertos que garanticen la mitigación de estos problemas generados a partir del estudio.

\section{RESULTADOS}

El Análisis e interpretación de los datos recopilados, permitieron llegar a las siguientes apreciaciones:

- Identificación de variables se puede observar en la Tabla 1.

Tabla 1. Identificación de variables que inciden en la baja productividad de las diferentes actividades de obra en Ocaña.

\begin{tabular}{|c|c|}
\hline No. & Variable \\
\hline & \\
\hline 1 & Economía General \\
\hline 2 & Aspectos laborales \\
& \\
\hline 3 & Clima \\
\hline 4 & Actividad \\
\hline
\end{tabular}

\begin{tabular}{|c|c|}
\hline 5 & \\
\hline 5 & Equipamiento \\
\hline 6 & Supervisión \\
\hline 7 & Trabajador \\
\hline 8 & Trabajo \\
\hline 10 & Pérdida de tiempo productivo \\
\hline 9 & Administración de la obra \\
\hline & \\
\hline & \\
\hline
\end{tabular}

Fuente. Elaboración propia.

Para determinar la población de las seis obras en estudio se realiza una clasificación de encuestados y se hace un censo que se puede observar en la Tabla 2.

Tabla 2. Recurso humano empleado en las diferentes obras de estudio de acuerdo a censo.

\begin{tabular}{|c|c|c|}
\hline Maestros & $\begin{array}{c}\text { Oficiales de } \\
\text { construcción }\end{array}$ & $\begin{array}{c}\text { Obreros de } \\
\text { construcción }\end{array}$ \\
\hline 6 & 9 & 70 \\
\hline
\end{tabular}

Fuente. Elaboración propia.

Para tomar un número suficiente de encuestados y de acuerdo a experiencias investigativas se tomó para el desarrollo de la misma una formula estadística para una 
población finita (1) y cuyo resultado se muestra a continuación:

$$
\begin{aligned}
& n=\frac{85 * 1.96^{2} * 0.05 * 0.95}{0.05^{2} *(85-1)+1.96^{2} * 0.05 * 0.95} \\
& n=40 \text { encuestas. }
\end{aligned}
$$

Luego se elabora la encuesta tipo cerrada con una serie de preguntas en total once, donde se abordó una serie de cuestiones de acuerdo a los ítems causa de la baja productividad en actividades de obra en Ocaña, desarrollada la misma se procesa para medir la confiabilidad de esta, estos resultados se pueden apreciar en la Imagen 1.

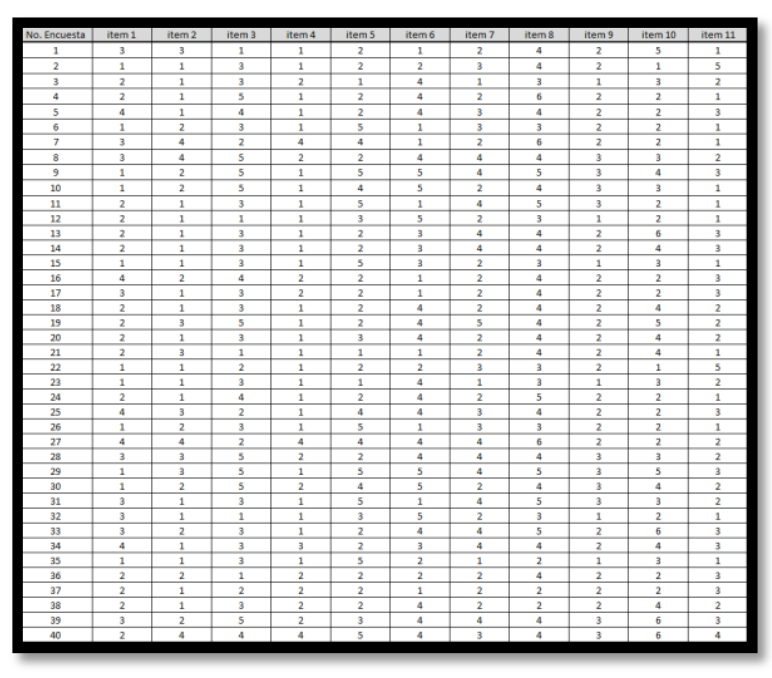

Imagen 1. Estructura organizativa para evaluar el coeficiente de alfa Combrash de acuerdo a encuestas realizadas.

Fuente. Elaboración propia.

Luego se evalúa con el coeficiente de alfa Combrash:

$$
\begin{aligned}
& \alpha=\left[\frac{11}{11-1}\right] *\left[1-\left(\frac{14.1}{46.26}\right)\right] \\
& \alpha=0.76
\end{aligned}
$$

De acuerdo a este resultado se puede concluir que la encuesta realizada es confiable, se encuentra dentro de los rangos de confiabilidad para este tipo de investigación.

Luego de validar la confiabilidad de la encuesta cerrada se procesan los datos, en la del proyecto se obtienen variables que inciden en la baja productividad para tomar las variables más representativas se toma un modelo estadístico aplicando el principio de Pareto, de acuerdo a este principio se evalúan los diferentes ítems causales de la baja productividad en las obras de Ocaña. En la Tabla 3 se puede observar el cuadro de frecuencias de acuerdo a las encuestas realizadas.

Tabla 3. Cuadro de frecuencias.

\begin{tabular}{|l|c|c|c|}
\hline $\begin{array}{l}\text { item (Variables } \\
\text { de estudio) }\end{array}$ & Frecuencia (f) & $\begin{array}{c}\text { Frecuencia } \\
\text { relativa (fr) }\end{array}$ & $\begin{array}{c}\text { Frecuencia } \\
\text { acumulada (fa) }\end{array}$ \\
\hline $\begin{array}{l}\text { Aspectos } \\
\text { laborales }\end{array}$ & 34 & $20,86 \%$ & $20,86 \%$ \\
\hline $\begin{array}{l}\text { Perdida de } \\
\text { tiempo } \\
\text { productivo }\end{array}$ & 26 & $15,95 \%$ & $36,81 \%$ \\
\hline Supervicion & 23 & $14,11 \%$ & $50,92 \%$ \\
\hline Trabajador & 20 & $12,27 \%$ & $63,19 \%$ \\
\hline Equipamiento & 18 & $11,04 \%$ & $74,23 \%$ \\
\hline $\begin{array}{l}\text { Administracion } \\
\text { de la obra }\end{array}$ & 14 & $8,59 \%$ & $82,82 \%$ \\
\hline Actividad & 13 & $7,98 \%$ & $90,80 \%$ \\
\hline Clima & 8 & $4,91 \%$ & $95,71 \%$ \\
\hline Trabajo & 5 & $3,07 \%$ & $98,77 \%$ \\
\hline Economia & 2 & $1,23 \%$ & $100,00 \%$ \\
\hline
\end{tabular}

Fuente. Elaboración propia.

De acuerdo a esta Tabla 3 se construye el diagrama de Pareto para determinar las variables de mayor incidencia en el estudio, se puede apreciar en la Imagen 2. 


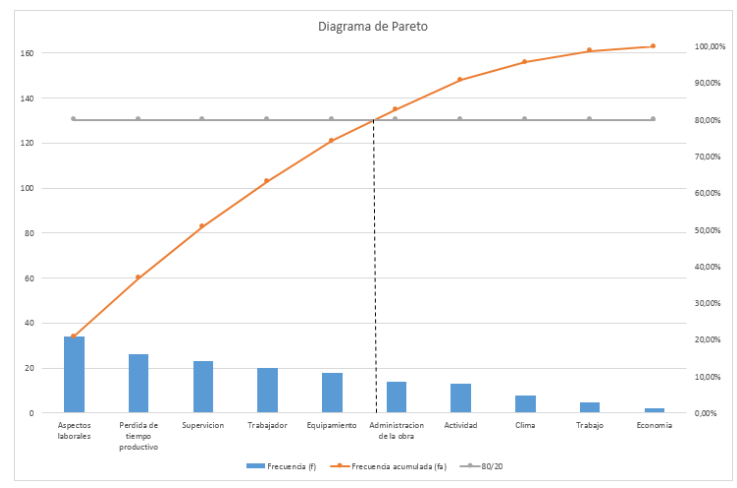

Imagen 2. Principio de Pareto aplicado a las variables de estudio.

Fuente. Elaboración propia.

De acuerdo a este principio cuyo resultado muestra las variables de mayor incidencia en la baja productividad en algunas actividades de obra en Ocaña las cuales se detallan en la Tabla 4.

Tabla 4. Principio de Pareto aplicado a las variables de estudio.

\begin{tabular}{|c|}
\hline $\begin{array}{c}\text { Variables de mayor influencia } \\
\text { en la baja productividad en } \\
\text { diferentes obras de } \\
\text { construcción en Ocaña }\end{array}$ \\
\hline Aspectos laborales \\
\hline Pérdida de tiempo productivo \\
\hline Supervisión \\
\hline Trabajador \\
\hline Equipamiento \\
\hline
\end{tabular}

Fuente. Elaboración propia.

El principio dice que el $20 \%$ de las variables en estudio representadas en la Tabla 4 sus acciones producirá el $80 \%$ de los efectos, mientras que el $80 \%$ restante sólo origina el $20 \%$ de estas variables representativas.

De acuerdo al principio de Pareto las principales variables que causan los problemas de baja productividad en las diferentes obras de construcción en Ocaña se pueden observar en la Tabla 4, para determinar las sub causas y con juicio de expertos se emplea el diagrama de Ishikawa; cuyo resultado se muestra en la Imagen 3, donde se analizan las posibles sub causas que generan estos problemas para tomar acciones que permitan a constructores de la región tomar medidas adecuadas para mitigar esta problemática.

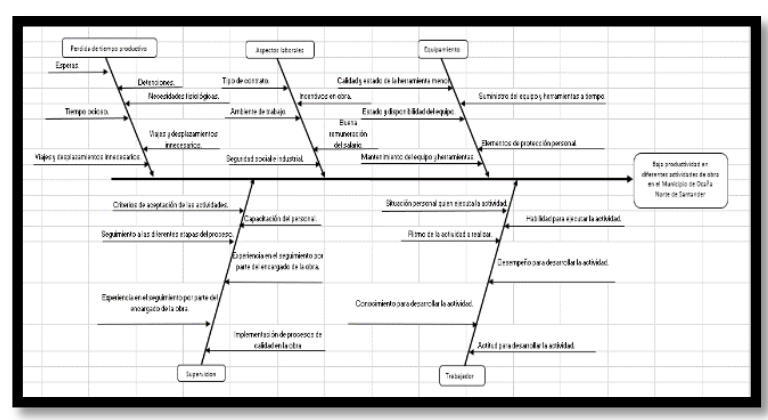

Imagen 3. Diagrama de ISHIKAWA para identificar las posibles sub causas derivadas de las causas potenciales.

Fuente. Elaboración propia.

Esta representación gráfica se organiza de forma lógica $y$ en orden de mayor importancia las causas potenciales las cuales son las representadas en la Tabla 4, estas contribuyen a crear un efecto o problema, también se detallan las posibles sub causas que generan estas causas potenciales.

\section{CONCLUSIONES}

- Dentro del estudio se pudo detallar que una de las variables que tienen mayor incidencia en la baja productividad corresponden a los aspectos laborales y es la que tiene mayor representación, esto se debe al tipo de contratación realizada al personal que labora en las diferentes construcciones, otras causas que generan esta problemática no hay 
buena remuneración del salario por parte de los que ejecutan la obra no se paga lo justo sino hace que el ambiente en las obras sea desmotivador haciendo que el obrero baje notablemente su rendimiento; el $85 \%$ de la población encuestada afirma que el contrato no es el adecuado a esto se suma que no hay incentivos dentro de la obra para mejorar este aspecto.

- Otras de las variables de mayor incidencia correspondió a la pérdida de tiempo productivo en desarrollar las actividades de obra; el $65 \%$ de la población encuestada afirman que hay mucha perdida de tiempo en desplazamiento de materiales y equipo para desarrollar las actividades, que en las obras los retrazos de los materiales al traerlos a la misma es eminente a esto sumado no se cuenta con lo suficientes recursos en el momento de ejecutar la actividad; como medida par mitigar esta causa las empresas constructoras y constructores de la ciudad deberian de desarrollar un plan de gestion de adquisiciones con cronograma de recursos del proyecto, que se enlazara a las actividades programadas en la obra; el tiempo ocioso fue otra de las variables que influyen en este aspecto los encuestados afirma que en las obras los tiempos donde no se labora "tiempo no productivo" se pierde mucho donde los obreros desarrollan actividades que no tienen nada que ver con la actividad. En este proceso se deberia de desarrollar un seguimiento al tiempo empleado por el personal para controlar mas este tiempo no productivo.
- Otra variable fue la supervisión; el $57,7 \%$ de los encuestados afirman que el personal para desarrollar las actividades es poco capacitada para hacer una actividad, no se cuenta con planes de capacitación dentro de las obras para ejecutar la acción, la estrategia seria desarrollar planes utilizando entidades como el SENA donde se capacite y certifique la mano de obra.

- Otra variable fue Trabajador; el 50\% de la población encuestada afirman que algunos obreros carecen de conocimiento para desarrollar la actividad a esto se suma la otra variable relevante el desempeño para realizarla la carecia de conocimiento hace que el desempeño no sea el mas optimo, el trabajo se retraza de manena considerable por parte del trabajador que desarrolla la actividad, para mitigar esta causa se deberia de contratar mas personal de mano calificada en las obras de construccion con el fin de corregir este flagelo.

- Otra variable fue Equipamiento; el $45 \%$ de la población encuestada afirma que los elementos de proteccion son insuficientes sobre todo cuando se trabaja en alturas poniendo en riesgo la vida del obrero, la calidad y estado de la herramienta usado para las diferentes actividades, se pudo notar en las obras visitadas la carencia de equipo $\mathrm{y}$ herramienta en buen estado, los contratistas no dotan los obreros adecuadamente con este recurso, se debe crear políticas dentro del proyecto de obra que asegure el desarrollo de la actividad con 
excelentes equipos y herramientas de calidad óptima y que se genenera una cultura sbre el aplicativo de normas y reglamentos para la proteccion $\mathrm{y}$ seguridad industrial en los proyectos de construccion en nuestra ciudad.

\section{FINANCIACIÓN}

El desarrollo del proyecto de investigación que arrojó como resultado este artículo es "Estudio de variables que inciden en la baja productividad de la mano de obra en diferentes actividades de construcción en Ocaña Norte de Santander". Afortunadamente este trabajo fue respaldado por el Grupo de Investigación en Geotecnia y Medio Ambiente (GIGMA), y con el apoyo financiero de la Universidad Francisco de Paula Santander.

\section{BIBLIOGRAFÍA}

Borcherding, J. D. (1976). Managerial Strategies for More Effective Utilization of Human Resources. Journal of the American Professional Constructor.

Hazeltine, C. S. (1976). Motivation of Construction Workers. Journal of the Construction Division, American Society of Civil Engineers.

Ishikawa, K. (1986). ¿Qué es control total de la calidad? Normal.

López de Ortigosa Casares, D. A. (2010). Ingeniería de costos en la construcción. México: Trillas.
Maslow, A. H. (1954). Motivation and Personality. Nueva York: Harper and Row Publishers.

McGregor, D. (1960). The Human Side of Enterprise. Nueva York: McGrawHill.

Monje Álvarez, C. A. (2011).

Metodología de la Investigación Cualitativa y Cuantitativa. Neiva: Universidad Surcolombiana.

Schrader, C. R. (1972). Motivation of Construction Craftsmen. Journal of the Construction División, American Society of Civil Engineers.

Tucker, R. L. (1988). Perfection of the Buggy Whip. Journal of the construction Engineering and Management, American Society of Civil Engineers, 158-171. 